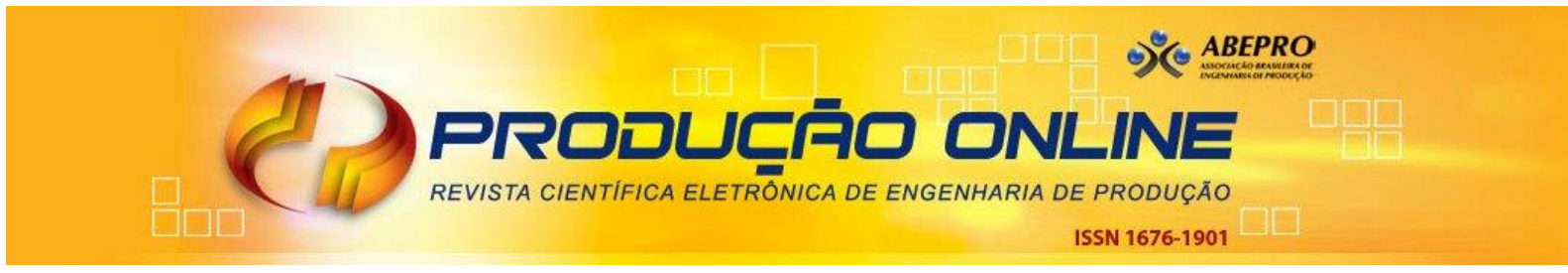

\title{
AUMENTO DO DESEMPENHO FABRIL SOB A LUZ DA TEORIA DAS RESTRIÇÕES: O CASO DE UMA FÁBRICA DE COLCHÕES
}

\section{INCREASED MANUFACTURING PERFORMANCE IN THE LIGHT OF THE THEORY OF CONSTRAINTS: THE CASE OF A MATTRESS FACTORY}

\author{
André Luiz Emmel Silva*E-mail: andresilva@unisc.br \\ Vicente Cunha da Silva*E-mail: vicente 027@hotmail.com \\ Liane Mahlmann Kipper*E-mail: liane@unisc.br \\ Fernando Sansone de Carvalho*E-mail: fernandocarvalho@unisc.br \\ Jorge André Ribas Moraes* E-mail: jorge@unisc.br \\ *Universidade de Santa Cruz do Sul (UNISC), Santa Cruz do Sul, RS, Brasil
}

\begin{abstract}
Resumo: A Teoria das Restrições (TOC) possibilita identificar, controlar e gerenciar da melhor maneira os gargalos em qualquer sistema produtivo por meio de cinco passos para o gerenciamento das restrições. O objetivo do presente trabalho é aumentar o desempenho do processo produtivo com a eliminação dos gargalos através da aplicação da Teoria das Restrições e das diretrizes dos seus cinco passos de focalização em uma indústria de colchões situada no Rio Grande do Sul. A pesquisa foi realizada com cunho objetivo exploratório e descritivo à Teoria das Restrições, pesquisa-ação com intervenções e ainda outras técnicas de avaliação, análise e identificação para melhoria no processo, em que se apresentam como ferramentas de auxílio. Os resultados encontrados relacionam-se com a identificação dos gargalos, noção do processo gargalo e sua eliminação. Na etapa 2 da TOC 1 (realizada no setor de revisão e embalagem), a quantidade de peças por hora aumentou de 43 para 51 o que significa $18,6 \%$, onde posteriormente na etapa 4 de 51 para 72 peças, ou seja, um aumento de $41 \%$, em decorrência da compra de um embaladora automática, sendo que ainda nesta TOC, foi possível a redução de 5 para 3 funcionários. Com a TOC 2 (realizada no setor de montagem) a evolução de peças foi de 59 para 71 , cerca de $24 \%$, devido as mudanças de layout e fluxo de produção contando ainda com a redução de 17 para 11 funcionários no setor. Com os resultados obteve-se ganhos significativos em produtividade, melhorando-se assim a performance da unidade fabril.
\end{abstract}

Palavras-chave: Teoria das restrições. Gargalo. Melhoria contínua. Indústria de colchões

\begin{abstract}
The Theory of Constraints (TOC) makes possible to identify, control and manage gaps in the best way possible at any productive system by using five steps to do the restrictions management. The goal of this work is to increase the productive process performance with the exclusion of gaps through the application of the Theory of Constraints and the guidelines of the focalization's five steps in a mattress factory located at Rio Grande do Sul. The research was done with an exploratory objective and descriptive nature to the Theory of Constraints, action research with interventions and also other evaluation techniques, analysis and identification to the process improvement, where they show themselves as supporting tools. The results relate to the identification of gaps, gaps process notion and its elimination. With the TOC 1 (held in the packaging sector and review) the amount of parts per hour increased from 43 to 51 with the step 2, which is equivalent to $18,6 \%$, and after in the step 4 from 51 to 72 parts, a $41 \%$ increase by the purchase of an automatic packing machine, also in this TOC was possible a reduction of employees from 5 to 3 . With the TOC 2 (held in the assembly sector) the increase of the parts amount was from 59 to 71 , about $25 \%$, by the layout and production flow changes, counting with the employees reduction from 17 to 11 in the sector. With the results there was significant production gains, increasing by that this factory unit performance.
\end{abstract}


Keywords: Theory of constraints. Bottleneck. Continuous improvement. Mattress industry.

\section{INTRODUÇÃO}

Para que uma empresa gere lucro e obtenha sucesso, o seu desempenho organizacional necessariamente deve ser eficiente. Percebe-se que é vital o gerenciamento dos seus custos e despesas para que os resultados sejam alcançados. Diante disso é preciso observar a maneira em que são utilizados os recursos disponíveis e que movimentam a organização, ainda que estes sejam fundamentais para qualificar sua competitividade no mercado cada vez mais acirrado. Esse cenário favorece a aplicação da Teoria das Restrições - TOC (INMAN, SALE e GREEN JR, 2009), que atua como protagonista para o aumento do desempenho fabril, deixando o ambiente produtivo inquieto e provocativo em busca de constantes oportunidades de melhoria.

A TOC faz com que os gestores concentrem-se nos pontos críticos do processo (WU, LEE e TSAI, 2014), enfatizando a importância do gargalo de uma forma estruturada e abrangente, e não investindo (recursos, esforços) naquilo que não agrega valor (OLIVEIRA, MARINS e ALMEIDA, 2010). É uma filosofia gerencial que proporciona aumento de ganho, redução dos níveis de inventário e de despesa operacional, melhorando assim a performance organizacional (BOYD e GUPTA, 2004; PACHECO, 2014).

Sendo assim, o presente trabalho teve por objetivo aumentar o desempenho do processo de fabricação de colchões de mola, modelo casal, da empresa em estudo, por intermédio da Teoria das Restrições. Em sua aplicação, utilizou-se a cronoánalise como ferramenta inicial, servindo como base de apoio, onde os tempos de cada atividade do sistema produtivo foram levantados e utilizados para as tomadas de decisão. A concepção da TOC na unidade fabril tornou-se importante não só para o ganho apresentado neste trabalho, mas também para estruturar os processos de focalização das cinco etapas constantemente, o que considerou-se fundamental para que, de fato, a melhoria contínua venha a sobreviver. Ganhos em volume de produção, redução de custos, investimentos em tecnologia, e percepção dos recursos que agregam valor estão demonstrados neste trabalho.

O trabalho está estruturado em cinco capítulos. Na Seção 2 apresenta-se o referencial teórico, com enfoque para a Teoria das Restrições e suas etapas, e o 
estudo de tempos e movimentos; na Seção 3 é apresentado o método e classificação da pesquisa; na Seção 4 está a descrição do problema e análise dos resultados; na Seção 5 está a conclusão do trabalho; e finalmente, ao final, apresenta-se as referências bibliográficas utilizadas no texto.

\section{FUNDAMENTAÇÃO TEÓRICA}

Nessa seção são apresentadas as bases teóricas que fundamentam esse trabalho: i) Teoria das Restrições; e ii) Estudo de tempos e movimentos. Também é apresentada uma bibliometria sobre a Teoria das Restrições no contexto atual.

\subsection{Teoria das Restrições}

A Teoria das Restrições (Theory of Constraints - TOC) tem sua origem a partir do aperfeiçoamento do software OPT (Optimized Production Technology) idealizado por Eliyahu Goldratt (NAOR, BERNARDES e COMAN, 2013). Porém, sua grande difusão ocorreu no início da década de $80 \mathrm{com}$ lançamento do livro A Meta (BLACKSTONE, 2001). Ela proporciona um caminho para compreender as organizações, em especial o seu processo produtivo essencial, visualizando a empresa não em partes isoladas, mas como um sistema integrado ou um conjunto de elementos entre os quais há algum tipo de ligação (QUELHAS e BARCAUI, 2008). É uma filosofia de gestão integrada que muda a maneira de pensar dos gestores e tornando-se uma importante ferramenta para a solução de problemas (ŞIMŞITA, GÜNAYB, VAYVAY, 2014).

Ao analisar esta teoria, encontrou-se a metodologia dos "cinco passos", onde, de forma estruturada e simplificada, se consegue executar ciclos da TOC em um processo visando elevar a restrição. Uma bibliometria quantitativa nas bases de dados Scopus (Elsevier) e Web of Science, em artigos, com o intuito de analisar a ocorrência de pesquisas embasadas na metodologia dos cinco passos da TOC para identificar os gargalos do processo e promover melhoria contínua foi realizada a fim de construir um panorama da evolução acadêmica destes temas. Pesquisando-se as palavras-chave "Theory of Constraints", "Bottleneck", "Continuous improvement" e "Five Focusing Steps" nos últimos dez anos nas bases de dados Scopus (Elsevier) e 
Web of Science encontrou-se um número de artigos publicados distribuídos de acordo com os quadros 1 e 2 .

Quadro 1 - Distribuição de artigos e temas relacionados na Base Scopus (Elsevier) 2005-2015

\begin{tabular}{|c|c|c|c|c|}
\hline $\begin{array}{c}\text { Scopus (Elsevier) } \\
\mathbf{2 0 0 5 - 2 0 1 5}\end{array}$ & $\begin{array}{c}\text { Theory of } \\
\text { Constraints }\end{array}$ & Bottleneck & $\begin{array}{c}\text { Continuous } \\
\text { improvement }\end{array}$ & $\begin{array}{c}\text { Five Focusing } \\
\text { Steps }\end{array}$ \\
\hline $\begin{array}{c}\text { Theory of } \\
\text { Constraints }\end{array}$ & 412 & 80 & 23 & 12 \\
\hline $\begin{array}{c}\text { Bottleneck } \\
\text { Continuous } \\
\text { improvement }\end{array}$ & & 18.161 & 24 & 04 \\
\hline Five Focusing Steps & & 3.772 & 05 \\
\hline
\end{tabular}

Quadro 2 - Distribuição de artigos e temas relacionados na Base Web of Science, 2005-2015

\begin{tabular}{|c|c|c|c|c|}
\hline $\begin{array}{c}\text { Scopus (Elsevier) } \\
\mathbf{2 0 0 5 - 2 0 1 5}\end{array}$ & $\begin{array}{c}\text { Theory of } \\
\text { Constraints }\end{array}$ & Bottleneck & $\begin{array}{c}\text { Continuous } \\
\text { improvement }\end{array}$ & $\begin{array}{c}\text { Five Focusing } \\
\text { Steps }\end{array}$ \\
\hline $\begin{array}{c}\text { Theory of } \\
\text { Constraints }\end{array}$ & 177 & 33 & 11 & 05 \\
\hline Bottleneck & & 10983 & 16 & 01 \\
\hline $\begin{array}{c}\text { Continuous } \\
\text { improvement }\end{array}$ & & 1739 & 04 \\
\hline Five Focusing Steps & & & & 06 \\
\hline
\end{tabular}

Da análise dos quadros 1 e 2 observa-se um número pequeno de publicações que descrevem as cinco etapas de focalização (Five Focusing Steps), sendo 06 encontradas na base Web of Science e 13 na base Scopus (Elsevier). Isto revela a necessidade de explorar de forma teórica e prática este tema. Comparando os artigos encontrados nas duas bases para esta palavra-chave observou-se que os seis artigos encontrados pela base Web of Science também aparecem no conjunto dos 13 artigos encontrados Scopus (Elsevier). Também observamos que um artigo estava duplicado na mesma base, sendo assim, apenas 12 artigos que tratam das cinco etapas de focalização foram encontrados no período de 2005 - 2015. Comparando a distribuição de artigos contendo uma palavra-chave com os resultados encontrados nos cruzamentos entre as palavras-chave observa-se um grande número de artigos contendo apenas uma das palavras-chave em detrimento aos cruzamentos ocorridos. Isto demonstra que a Teoria das Restrições (TOC) 
mesmo tendo uma grande divulgação desde a década de 80, ainda encontra-se presente nas publicações acadêmicas. No quadro 3 apresentam-se os 06 artigos encontrados nas duas bases de periódicos.

Quadro 3 - Artigos citados nas duas bases de periódicos pesquisadas

\begin{tabular}{|c|c|c|c|c|}
\hline $\begin{array}{c}\text { Autor(es)(a } \\
\text { no) }\end{array}$ & Periódico & Título & Objetivo & $\begin{array}{c}\text { Principais resultados } \\
\text { encontrados }\end{array}$ \\
\hline $\begin{array}{l}\text { Umble, et al. } \\
\quad(2006)\end{array}$ & $\begin{array}{l}\text { International } \\
\text { Journal of } \\
\text { production } \\
\text { research }\end{array}$ & $\begin{array}{l}\text { Implementing } \\
\text { theory of } \\
\text { constraints in a } \\
\text { traditional } \\
\text { Japanese } \\
\text { manufacturing } \\
\text { environment: the } \\
\text { case of Hitachi Tool } \\
\text { Engineering }\end{array}$ & $\begin{array}{l}\text { Aplicar a TOC } \\
\text { em uma } \\
\text { fábrica } \\
\text { japonesa de } \\
\text { ferramentas. }\end{array}$ & $\begin{array}{l}\text { A implementação apresentou } \\
\text { sucesso e gerou melhorias } \\
\text { significativas no estoque de } \\
\text { trabalho em processo, tempo } \\
\text { de produção, entrega no } \\
\text { prazo, capacidade produtiva, } \\
\text { rotação de estoque, qualidade } \\
\text { do produto, volume de vendas } \\
\text { e lucratividade. } \\
\text { A TOC está se tornando a } \\
\text { cultura de empresa. }\end{array}$ \\
\hline $\begin{array}{l}\text { Gupta and } \\
\text { Kline (2008) }\end{array}$ & $\begin{array}{l}\text { Total Quality } \\
\text { Management } \\
\text { \& Business } \\
\text { Excellence }\end{array}$ & $\begin{array}{c}\text { Managing a } \\
\text { community mental } \\
\text { health agency: } A \\
\text { Theory of } \\
\text { Constraints based } \\
\text { framework }\end{array}$ & $\begin{array}{c}\text { Aplicar a TOC } \\
\text { nos sistemas } \\
\text { de prestação } \\
\text { de serviços de } \\
\text { saúde. }\end{array}$ & $\begin{array}{c}\text { A adoção de uma abordagem } \\
\text { da TOC para gestão } \\
\text { de operações melhorou a } \\
\text { gestão operacional e financeir } \\
\text { o da unidade estudada. }\end{array}$ \\
\hline $\begin{array}{l}\text { Dalton } \\
\text { (2009) }\end{array}$ & $\begin{array}{l}\text { Research- } \\
\text { Technology } \\
\text { Management }\end{array}$ & $\begin{array}{c}\text { What's } \\
\text { Constraining Your } \\
\text { Innovation? }\end{array}$ & $\begin{array}{c}\text { Explicar a } \\
\text { Teoria das } \\
\text { Restrições } \\
\text { (TOC), } \\
\text { relacionando } \\
\text { com a cultura } \\
\text { de melhoria } \\
\text { contínua da } \\
\text { inovação. }\end{array}$ & $\begin{array}{l}\text { Apresenta os cinco passos de } \\
\text { focalização são utilizados para } \\
\text { eliminar rigorosamente essa } \\
\text { restrição e avançar para a } \\
\text { próxima com um aumento no } \\
\text { rendimento da inovação à } \\
\text { medida que cada ciclo de } \\
\text { melhoria é completado. }\end{array}$ \\
\hline $\begin{array}{l}\text { Gupta et al. } \\
\quad(2010)\end{array}$ & $\begin{array}{l}\text { Total Quality } \\
\text { Management } \\
\text { \& Business } \\
\text { Excellence }\end{array}$ & $\begin{array}{c}\text { A TOC-based } \\
\text { framework for small } \\
\text { businesses }\end{array}$ & $\begin{array}{l}\text { Apresentar os } \\
\text { 3Ms da TOC } \\
\text { de forma } \\
\text { simples para } \\
\text { uso nas } \\
\text { pequenas } \\
\text { empresas. }\end{array}$ & $\begin{array}{l}\text { Na empresa estudada a TOC } \\
\text { melhorou os processos de } \\
\text { produção, aumentando } \\
\text { continuamente a produção de } \\
\text { qualidade superior e } \\
\text { consistente resultando em um } \\
\text { alto nível de desempenho. O } \\
\text { foco da aplicação foi na } \\
\text { produção, menores estoques e } \\
\text { despesas operacionais (nessa } \\
\text { ordem) A mentalidade TOC se } \\
\text { concentra em ganhar dinheiro } \\
\text { em vez de poupar dinheiro, e } \\
\text { garante a segurança dos } \\
\text { funcionários e satisfação do } \\
\text { cliente. }\end{array}$ \\
\hline
\end{tabular}


Quadro 3 - Artigos citados nas duas bases de periódicos pesquisadas

\begin{tabular}{|c|c|c|c|c|}
\hline $\begin{array}{c}\text { Autor(es)(a } \\
\text { no) }\end{array}$ & Periódico & Título & Objetivo & $\begin{array}{c}\text { Principais resultados } \\
\text { encontrados }\end{array}$ \\
\hline $\begin{array}{c}\text { Lenort, } \\
\text { Klepek and } \\
\text { Samolejová } \\
(2012)\end{array}$ & Metalurgija & $\begin{array}{l}\text { Heuristic Algorithm } \\
\text { for Planning and } \\
\text { Scheduling of } \\
\text { Forged Pieces Heat } \\
\text { Treatment }\end{array}$ & $\begin{array}{c}\text { Projetar um } \\
\text { algoritmo } \\
\text { heurístico para } \\
\text { planejamento } \\
\text { e } \\
\text { programação } \\
\text { de peças } \\
\text { forjadas na } \\
\text { busca de } \\
\text { maximizar } \\
\text { gargalos. }\end{array}$ & $\begin{array}{l}\text { A implementação do algoritmo } \\
\text { projetado no processo } \\
\text { pesquisado de tratamento } \\
\text { térmico trouxe um aumento de } \\
25 \% \text { na exploração da } \\
\text { capacidade e através da } \\
\text { colocação de todo o processo } \\
\text { de forjamento. }\end{array}$ \\
\hline $\begin{array}{l}\text { Pretorius } \\
(2014)\end{array}$ & $\begin{array}{l}\text { International } \\
\text { Journal of } \\
\text { Production } \\
\text { Research }\end{array}$ & $\begin{array}{l}\text { Introducing in- } \\
\text { between decision } \\
\text { points to TOC's five } \\
\text { focusing steps }\end{array}$ & $\begin{array}{l}\text { Aplicar as } \\
\text { cinco etapas } \\
\text { de focalização } \\
\text { incluindo } \\
\text { restrições não- } \\
\text { físicas, por } \\
\text { meio de } \\
\text { programação } \\
\text { linear. }\end{array}$ & $\begin{array}{c}\text { Desenvolvimento de um } \\
\text { diagrama de fluxo } \\
\text { demonstrando aplicabilidade } \\
\text { dos cinco passos da TOC a } \\
\text { todos os tipos de restrições, } \\
\text { independentemente de serem } \\
\text { restrições físicas ou não. }\end{array}$ \\
\hline
\end{tabular}

Observa-se na análise dos artigos que todos trazem em seu conteúdo uma explicação sobre a Teoria das Restrições (TOC) sendo considerada uma ferramenta com uso nas operações de fabricação e na identificação e eliminação de gargalos. Dalton (2009) comenta que a TOC e os cinco passos de focalização permitem aos inovadores melhorar rapidamente os seus resultados de crescimento e cria gradualmente uma cultura de melhoria contínua da inovação. Os artigos reforçam que uma abordagem TOC aplicada ao o processo atual para encontrar o gargalo - a restrição que está impedindo todas as outras etapas do processo pode promover melhorias, em especial com o uso dos cinco passos de focalização, eliminando rigorosamente essa restrição e avançando para a próxima promovendo ciclos de melhoria contínua.

Acrescenta-se ainda que a TOC pode ser compreendida como uma série de princípios teóricos, que resumem e fundamentam conhecimentos particulares de gestão e controle da produção, reconhecendo o papel dos fatores limitantes nas operações de manufatura e focando sobre os mesmos, tendo em vista o aumento ou o avanço de suas utilizações (SOBREIRO e NAGANO, 2013). O uso da TOC resulta em melhores índices dos processos, aumento na precisão das previsões e nos níveis de serviço ao cliente (WU et al., 2010), redução de inventários, prazos de entrega e tempos de produção (WATSON, BLACKSTONE, GARDINER, 2007). 
Conforme Goldratt e Cox (2003) o melhor modo de ilustrar qualquer sistema é por meio da relação de uma corrente, onde a resistência global da corrente é determinada pela força do seu elo mais fraco (restrição). Assim, restrição, ou gargalo, é algo que limita o desempenho do sistema, impedindo-o de atingir suas metas (MABIN e BALDERSTONE, 2003; ZIVALJEVIC, 2015). Pode ser um recurso físico, políticas gerenciais, ou ainda fatores comportamentais (BLACKSTONE, 2001).

Em determinadas circunstâncias, pode não haver gargalos reais em um centro produtivo da fábrica, pois todos os setores estão superdimensionados em relação à demanda, porém sempre existirá algum recurso que restrinja a produção (PRATES e BANDEIRA, 2011). Assim sendo, para que as empresas não caiam na problemática de produzir além do necessário, Goldratt e Cox (2003) expõem que o fluxo de produção deve ser conforme a demanda e não conforme a capacidade produtiva.

Todo esse processo de melhoria contínua se dá pelo esforço em abordar a restrição e trabalhar em seu benefício na eficiência da produção. Deste modo, a otimização acontece através das cinco etapas de focalização, onde é possível atacar apenas um determinado ponto que define o desempenho de todo o sistema. (GOLDRATT e COX, 2003).

\subsubsection{Etapa 1: Identificar a restrição do sistema}

Faz-se necessário identificar as possíveis restrições que impedem a empresa de atingir a sua meta (PERGHER, RODRIGUES e LACERDA, 2011). Qualquer sistema possui algo que limita seu crescimento, caso contrário, este cresceria sem limites e por onde pudesse passar iria consumir tudo (COX III e SPENCER, 2002). A identificação de um recurso-gargalo está inserida na essência de uma escala e tempo, ou seja, um recurso possui ou não capacidade suficiente de atendimento da demanda em uma determinada programação de produção (SOUZA, 2005).

\subsubsection{Etapa 2: Explorar a restrição do sistema}

Consiste em explorar a restrição no que tange a sua correta utilização, seus procedimentos e regras que muitas vezes não estão de acordo com o recurso, 
podendo gerar tempo perdido e influenciar no desempenho do sistema produtivo. $O$ fluxo deve-se manter e para isso uma das alternativas é verificar se está totalmente ocupado com os produtos certos de maneira que o lucro seja maximizado (COX III e SPENCER, 2002). Se a restrição for física, o objetivo é fazê-la tão eficaz quanto possível. Já a restrição gerencial deve ser eliminada e substituída por uma outra que viabilize o processo (BOSCHETTO et al, 2009). Alguns fatores podem ser avaliados para que o recurso seja explorado, tais como o tempo de preparação, tempo de setup, a eliminação de defeitos de fabricação, manutenções preventivas, limpezas, paradas para o almoço. Todos estes fatores contribuem para que o recurso se conforme a ser uma restrição caso não seja gerenciado. É necessário planejar o recurso de forma a ocupar, da mais perfeita maneira possível a disponibilidade do tempo produtivo, sabendo sempre da importância dos prazos de entrega dos pedidos (SOUZA, 2005). Ou seja, deve-se extrair o máximo deste recurso

\subsubsection{Etapa 3: Subordinar os demais processos à restrição}

As atividades de todo o sistema devem ser subordinadas à restrição encontrada no primeiro passo (PERGHER, RODRIGUES e LACERDA, 2011). 0 recurso com restrição de capacidade deve ser o responsável pelo rítmo de todo o fluxo de produção (CALIA e GUERRINI, 2005). Os recursos devem ser utilizados na medida exata, demandados pela restrição, o que caracteriza esta etapa como a mais difícil. Um problema será criado se os indicadores do recurso não-gargalo não estiverem ao encontro da restrição, pois todas as atividades devem ser direcionadas para tal, de forma que um líder de setor possa acreditar que suas máquinas e pessoal estão ociosos, o que é equivocado (COX III e SPENCER, 2002). A soma dos ótimos locais não é igual ao ótimo global (GOLDRATT e COX, 2003), ou seja, o processo por inteiro tem que evoluir.

\subsubsection{Etapa 4: Elevar a restrição}

Esta etapa é dada se há a necessidade de elevar. Quando o gargalo for interno é fundamental aumentar a capacidade produtiva, com mudanças de layout, diminuição da variabilidade, redução de setup ou aquisição de equipamentos. Porém 
a restrição pode ser externa, onde o mercado não compra, implicando em novas estratégias de venda, novos clientes, ou inovação de produtos (PACHECO, 2012). Cox III e Spencer (2002) descrevem que este passo é preciso erguer a restrição. Diferente da $2^{2}$ etapa onde se deve aproveitar ao máximo, esta se diferencia por elevar a restrição, sejam em colocar mais equipamentos, ou modificar os já existentes, como também, criar outros turnos de trabalho, acrescentar velocidade através de automatização, e utilização de mais mão de obra.

\subsubsection{Etapa 5: Voltar ao passo um e identificar uma nova restrição}

Torna-se necessário retornar à primeira etapa e identificar a próxima restrição, de modo que a inércia não tome conta do sistema, fechando o ciclo da melhoria contínua. De acordo com Cox III e Spencer (2002), o desempenho das relações humanas muda, os projetos de engenharia mudam, as decisões produzir versus comprar mudam, as demarcações de preços e de compostos de produtos os força para melhorar a qualidade e precisam ser estruturados em função da nova restrição.

\subsection{Estudo de tempos e movimentos}

Estudos de tempos e movimentos foram originalmente desenvolvidos na Engenharia Industrial e incidiram sobre a análise dos movimentos de uma tarefa com ênfase na quantidade de tempo necessário para realizá-la (LOPETEGUI et al, 2014). Requerem uma observação independente e contínua e são um método mais preciso do que o auto-relato ou técnicas de amostragem trabalho, que coleta dados em intervalos de tempo (COSTER et al, 2015). É definido como o estudo sistemático dos sistemas de trabalho com o objetivo de projetar o melhor método de trabalho, geralmente o de menor custo, padronizar este método e determinar o tempo gasto por uma pessoa qualificada e devidamente treinada, trabalhando em ritmo normal, para executar uma operação específica (CONTADOR, 1998). Possibilita racionalização da utilização de mão de obra, além da determinação do tempo padrão, a eliminação de desperdícios e excesso de movimentos (CHIAVENATO, 2003). 
Uma ferramenta importante para o estudo de tempos e movimentos é a cronoanálise, sendo uma forma de medir o trabalho e alcançar tempos padrões para as atividades de uma produção. Segundo Sugai (2003), a cronoanálise tem sua origem nos estudos de Taylor e Gilbreth, onde o primeiro deu enfoque à capacidade do operador, avaliando o processo produtivo por suas divisões, de maneira em que o segundo destacou o estudo em razão dos movimentos desnecessários e a fadiga do operador.

A utilização desta técnica aborda o fluxo de produção, o que permite identificar as necessidades do operador, quebras de maquinário, e outros fatores que geram desperdício de tempo. De acordo com Toledo Junior (1995), a cronoanálise é utilizada para definir o tempo padrão de uma tarefa que o colaborador execute em condições normais de trabalho. Alguns fatores são imprescindíveis para a realização do trabalho, como a velocidade do movimento, esforço, consistência, destreza, e devem ser considerados separadamente ou em combinação pelo observador para obtenção do cálculo do tempo utilizado (SLACK, CHAMBERS e JOHNSTON, 2002).

Outras duas ferramentas largamente utilizadas são a Pert (Program Evaluation and Review Technique) e a CPM (Critical Path Method), técnicas baseadas em rede que foram desenvolvidas no final da década de 50. A Pert foi desenvolvida para o projeto do míssil Polaris da Marinha norte-amaricana (RITZMAN e KRAJEWSKI, 2004), e a CPM foi criada para programar a construção, manutenção e desativação de fábricas de processos químicos (MOREIRA, 2013). Modelos em rede facilitam a visualização das relações entre os componentes do sistema, melhorando 0 entendimento do problema e seus possíveis resultados (LACHTERMACHER, 2009). Essas técnicas proporcionam um fórum para os gerentes de diversas áreas funcionais discutirem a natureza das várias atividades e suas necessidades de recursos (RITZMAN e KRAJEWSKI, 2004), tornando-se um instrumento valioso para o cálculo das necessidades de recursos em intervalos de tempo, confronto da disponibilidade com a necessidade e nivelamento dos recursos (ANDRADE, 2014). 


\section{MÉTODO E CLASSIFICAÇÃO DA PESQUISA}

Como forma de identificar o caráter metodológico dos trabalhos, Santos (2000) caracteriza as pesquisas segundo seus objetivos, segundo procedimentos de coleta, e segundo as fontes utilizadas na coleta de dados. O presente trabalho tem seu âmbito no objetivo exploratório e descritivo à Teoria das Restrições, e ainda outras técnicas de avaliação, análise e identificação para melhoria no processo, em que se apresentam como ferramentas de auxílio para a o estabelecimento das cinco etapas descritas na TOC.

No que se refere aos procedimentos de coleta, ocorreu a realização de uma revisão da literatura atual por meio de bibliometria, reconhecendo os princípios teóricos que desenvolveram a aplicação da TOC em diversas empresas, os resultados e sua contribuição para a melhoria contínua, como também sua evolução em diversos segmentos do mercado. Dos estudos bibliométricos foi analisada a atividade científica através de estudos quantitativos dos artigos publicados em periódicos internacionais, objetivou-se com este estudo entender a dinâmica atual do conhecimento sobre a Teoria das Restrições, bem como a importância desta para os pesquisadores em diversas áreas e organizações. Para isto foram pesquisadas as duas bases de periódicos mais importantes da atualidade, quais sejam, a Scopus (Elsevier) e a Web of Science (coleção completa) no período de 2005 - 2015.

Porém, a maior fonte de dados foi concebida pela pesquisa-ação, que possibilita $\mathrm{o}$ desenvolvimento dos processos envolvendo pesquisadores e pesquisados. Thiollent (2008) define como um tipo de pesquisa participante engajada com base empírica que é arquitetada e realizada em cooperação com uma ação ou com a resolução de um problema.

As extrações dos dados, como fonte de informação, foram produzidas entre bibliográficas e de campo. A bibliografia se fez necessária para a análise de dados, conclusões e raciocínios descritos por outros autores, para ampliar o conhecimento acerca do assunto e motivar o questionamento pessoal para novas ideias. A informação de campo, utilizada para perceber a rotina de trabalho do processo produtivo, os indicadores de produtividade por setor e a realidade do momento atual da empresa. 
Para atingir o objetivo a que se proponha, o trabalho seguiu as seguintes etapas: (i) avaliação dos tempos perdidos na restrição, através da aplicação da cronoanálise, com destaque para a identificação de atividades que não geram valor; (ii) estruturação do processo de focalização das cinco etapas, para gerenciar e controlar as restrições (gargalos) do processo; (iii) proposta do aumento de desempenho no componente restritivo, para que conseqüentemente acrescente-se velocidade ao sistema; e (iv) compreensão da eliminação das restrições e o surgimento de novas restrições ao sistema, sucessivamente, para que o processo de melhoria contínua permaneça existente.

\section{DESCRIÇÃO DO PROBLEMA E ANÁLISE DOS RESULTADOS}

No Brasil, a fabricação de colchões está integrada ao contexto do segmento moveleiro. Este setor é composto por inúmeras indústrias dos segmentos de madeira, metal e outros materiais em todo território nacional. A indústria brasileira de móveis está entre as mais admiráveis do segmento das Indústrias de Transformação no País, não só pelo valor da sua produção, mas também pela sua geração de empregos dentro da indústria nacional (CARVALHO, 2011).

Conforme fontes da Associação das Indústrias de Móveis do Estado do Rio Grande do Sul - MOVERGS, de 2014, no Rio Grande do Sul a indústria moveleira gaúcha é constituída por 2.500 empresas, das quais 86\% produzem móveis de madeira, $8,3 \%$ móveis de metal, $4,5 \%$ outros móveis e 1,2\% colchões. Este último índice representa a quantidade de 30 fábricas no estado.

A fabricação de colchões apresenta algumas particularidades, de acordo com os diferentes tipos existentes. Os colchões de espuma têm durabilidade menor que os colchões de mola, devido a sua flexibilidade. São compostos inteiramente por uma lâmina de espuma flexível de poliuretano, podendo ser classificado em diversas densidades (D18, D20, D28, D33, D45), cada um com características para se adequarem aos diversos biótipos do consumidor. A configuração de cada lâmina de espuma é resultado da reação química de Cloreto de Metileno (Diclorometano), Poliol Poliéster e Diisocianato de Tolueno (TDI), sendo estes os principais agentes, além de aditivos e outros materiais. Já os colchões de mola são compostos de uma camada menor de espuma, juntamente com molas distribuídas na área do colchão. 
São mais resistentes que os colchões de espuma e se adaptam facilmente a qualquer biótipo. Existe o modelo de molas ensacadas, onde o fio de aço é enrolado dentro de um TNT. Conhecido como "pockef", as molas trabalham em um sistema individual, deixando o colchão firme e com alta sustentação. Outro modelo é o chamado "bonnel”, em que o fio de aço não é ensacado mas é unido por arames. Este tem durabilidade menor que o "pocket" porque o conjunto de molas trabalha por inteiro. Qualquer impacto em um canto do colchão, afeta todo o restante. As molas trabalham em conjunto.

A pesquisa foi realizada em uma empresa fabricante de colchões localizada na região central do estado do Rio Grande do Sul, em uma área construída de 20mil metros quadrados. Produz anualmente cerca de 230 mil colchões de mola e espuma, 100 mil bases de madeira e 18 mil conjuntos de estofados. Suas atividades tiveram início no ano de 2007, e atualmente possui 270 funcionários. Ao longo dos seus 9 anos, muitos esforços foram realizados para que resultados lucrativos fossem atingidos. Neste aspecto o aprimoramento contínuo dos processos é destacado pelos gestores da unidade como fator principal, e oportunidades de melhoria devem ser provocadas constantemente. O período de análise foi de março a outubro de 2015.

Primeiramente, fez-se um levantamento do fluxo do processo (caracterizado por uma produção puxada) e dados de cronoanálise, evidenciados por meio de uma Rede PERT/CPM (Figura 1). As atividades que compõem o fluxo produtivo de colchões de mola, modelo casal são: A - Bordar rolo de faixa/pillow; B - Laminar rolo de faixa/pillow; C - Overlocar rolo de faixa/pillow; D - Bordar/Cortar tampo; (E) Overlocar tampo; (F) Montagem do caixote de molas; (G) Dobrar faixa e pillow Euro/Top; (H) Cortar pillow Top; (I) Costurar pillow Top; (J) Cortar faixa e pillow Euro; (K) Costurar faixa/Euro; (L) Montar colchão; (M) Costurar Viés; (N) Revisar colchão; e (O) Embalar colchão. O termo pillow no contexto em que está inserido significa uma camada extra, que fica por cima do colchão para deixá-lo mais macio. Overlocar é um processo de costura realizado nas bordas do colchão. 
Figura 1 - Rede PERT/CPM do processo

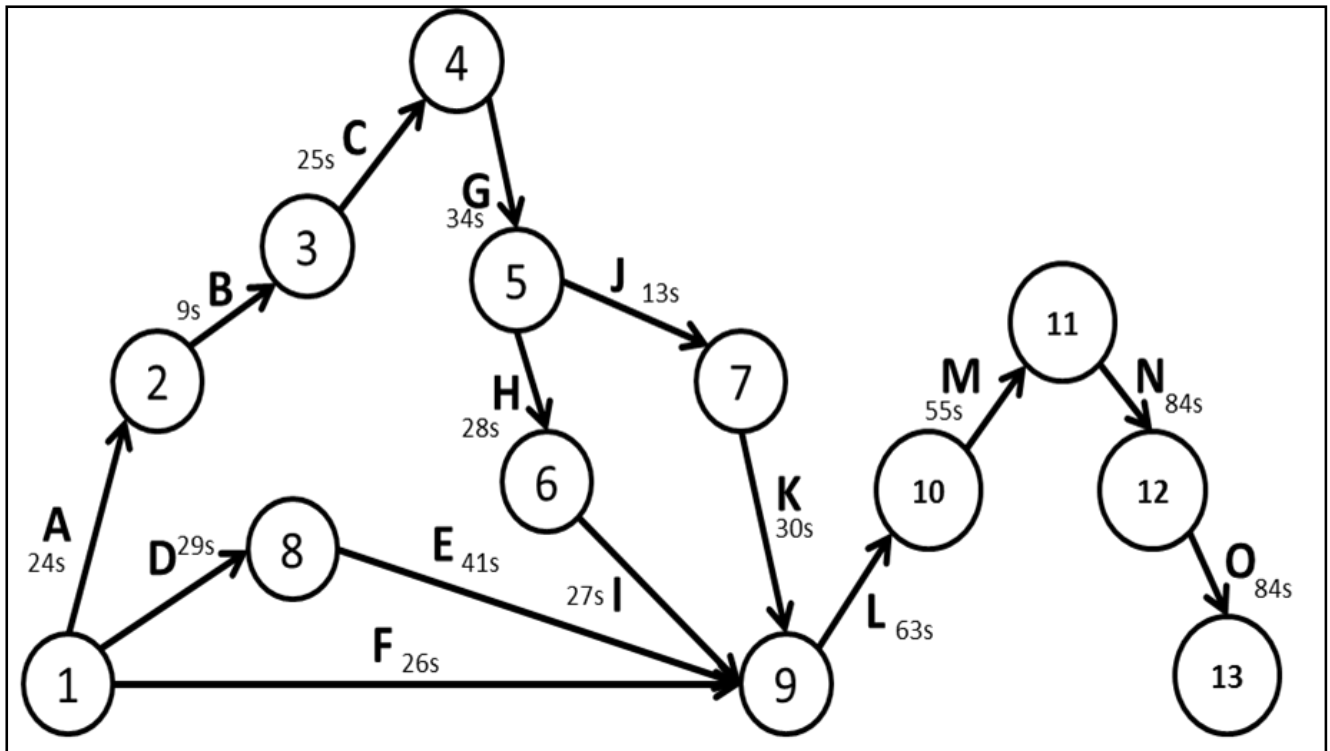

Fonte: Elaborado pelos autores

$\mathrm{Na}$ seqüencia, buscou-se aumentar o desempenho do processo produtivo através da aplicação das cinco etapas presentes na Teoria das Restrições: identificar, explorar, subordinar e elevar a restrição, obtendo como o último passo, voltar ao passo um, que é descobrir um novo gargalo. O primeiro giro das cinco etapas (TOC 1) ocorreu no setor de revisão e embalagem. Já o segundo giro (TOC 2) deu-se no setor de montagem do colchão.

\subsection{TOC 1 - Setor de revisão e embalagem}

Passo 1: Identificar a restrição do sistema - Após realizado levantamento de dados de cada setor de produção de colchões, foi constatado que o setor de revisão/embalagem (atividades $\mathrm{N}-\mathrm{O}$ na Figura 1) exercia uma baixa produtividade. São 5 funcionários exercendo suas funções em 2 mesas, 1 seladora por temperatura e 1 esteira transportadora, com uma produtividade de 43 peças por hora. Percebese que diariamente a esteira que leva o produto sempre se encontra cheia de colchões, de modo que os funcionários do setor de colchoaria (atividade $\mathrm{M}$ ) chegam a empilhar até 2 metros de altura, por falta de lugar para colocar o colchão. Quando se visualiza esta ocorrência de pilhas é notável acreditar que o processo está superdimensionado em relação ao setor revisão/embalagem (atividades $\mathrm{N}-\mathrm{O}$ na Figura 1), no qual não absorve a produtividade dos processos anteriores. 
Passo 2: Explorar a restrição do sistema - Durante três dias observou-se as atividades e seus respectivos tempos de produção, e encontrou-se três elementos que agregam valor ao processo e outros três que não agregam. Em virtude disso, relacionou-se por prioridade o item "retirar excessos de linha de costura", por consumir maior tempo do processo. Questionou-se junto aos líderes produtivos a ocasião de conter elevadas quantidades de linha para serem removidas, no qual foi mencionado de que a geração é dada parte pelo setor anterior (atividade M- Costurar viés na Figura 1) e parte por outros setores.

Em decorrência desta situação, adotou-se que as linhas em excessos geradas pela "atividade M", deveriam ser retiradas pelo próprio operador que gera esse excesso. Mesmo sabendo que o tempo do ciclo produtivo da "atividade M" iria aumentar efetuou-se esta mudança, pois se compreendeu que é mais fácil dividir tal elemento para outro setor (anterior e sem restrição) composto por 12 operadores do que manter o elemento exercido por 4 operadores de um setor identificado como gargalo.

Outra percepção foi que ao identificar uma não conformidade, a mesma era levada pelo próprio operador do setor de revisão/embalagem (atividades N-O) até o setor que gerou o problema, ou para o setor que pudesse consertá-lo gerando desperdício de tempo. Para tal fato realizou-se a implantação de uma área para produtos não-conformes, no próprio setor, para que o funcionário possa se desfazer o mais rápido possível, ficando para o setor de qualidade avaliar e destinar o conserto do produto. Como resultado da exploração do gargalo, a quantidade de peças processadas por hora aumentou de 43 para 51, um ganho percentual de $18,6 \%$.

Passo 3: Subordinar os demais processos à restrição - Conhecendo-se o novo valor de capacidade de produção nas atividades (N-O na Figura 1), proporcionou maior segurança na realização desta etapa de subordinação, pois todas as atividades anteriores foram programadas para este valor de peças por hora. Significou para a linha de produção a ociosidade de alguns equipamentos nas atividades $A, B, C$ e $D$ na Figura 1, onde os funcionários, multidisciplinares, passaram a atuar em mais de uma máquina. O reflexo deste cenário é de que a quantidade de estoque em processo reduziu a tal ponto de fazer estritamente o necessário. 
Passo 4: Elevar a restrição - Apesar dos resultados satisfatórios obtidos na exploração da restrição o setor ainda é considerado como gargalo, demandado por sua capacidade limitada e a oferta que outros setores proporcionam. Devido a isso, com a intenção de aumentar a capacidade produtiva do gargalo e atribuir tecnologia ao setor, percebeu-se a necessidade de melhorar os equipamentos, o fluxo e o layout do processo. Adquiriu-se de uma máquina automática de embalagem para colchões, passando de 71 segundos por peça para 50 segundos, um ganho percentual de $30 \%$. A quantidade de peças por hora elevou consideravelmente, chegando ao patamar de 72 peças por hora, cerca de $41 \%$ se comparado com a etapa 2.

Com a ampliação da área construída do complexo industrial motivou-se a alteração de layout das atividades $\mathrm{N}-\mathrm{O}$ para contribuir com a melhoria contínua do processo. A esteira que leva os colchões foi alocada ao sentido da máquina, ficando interligada com sistema de sensores automáticos, o que possibilitou ao setor retirar as 2 mesas de revisão e a máquina seladora por temperatura, pois a os elementos da atividade que compõem revisar o produto, são agora realizados na própria esteira. O fluxo do processo ganhou com isso, pois não há mais interrupções manuais em retirar os produtos da esteira, pois eles estão agora, alinhados e seguindo para a embalagem de maneira seqüencial. Outro efeito favorável é de que foi possível reduzir o efetivo de funcionários de cinco para três. A Figura 2 apresenta o layout com a esteira e a máquina após a etapa 4.

Figura 2 - Layout do setor de revisão e embalagem

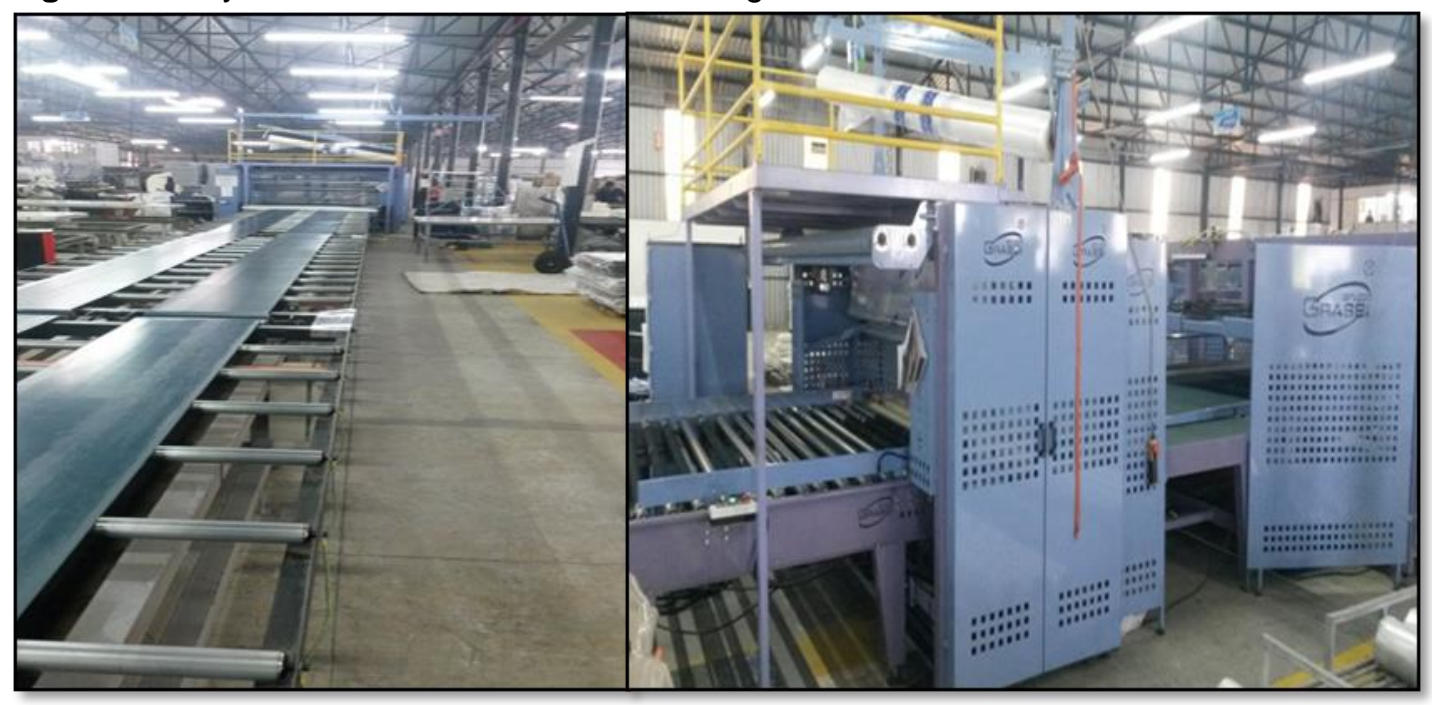

Fonte: Elaborado pelos autores 
Passo 5: Voltar ao passo um e identificar uma nova restrição - Foi possível constatar que a restrição encontrada no sistema foi quebrada e os percentuais de ganho demonstrados são essenciais para a melhoria contínua de toda a organização. Esse gargalo anteriormente era um impedimento "oculto" aos olhos da gestão da unidade, agora traz um papel diferente para a composição do sistema fabril. $O$ desempenho do componente restritivo é outro e novas análises devem ser feitas para identificar um novo gargalo, retornando ao passo 1.

\subsection{TOC 2 - Setor de montagem}

Passo 1: Identificar a restrição do sistema - Com a alteração de layout que foi descrita na TOC 1, a atividade L (montar o colchão na Figura 1) precisou ser dimensionada novamente, pois sua média de produção é de 63 segundos por peça, caracterizando-a como o novo gargalo. O setor conta com 7 mesas de fabricação, 4 coladeiras e 17 funcionários. O maior tempo improdutivo é levar o colchão montado para o setor de colchoaria (atividade $M$ na Figura 1). Através do histórico da cronoánalise cada funcionário perde cerca de 60 segundos para percorrer a distância de levar cada colchão fabricado até os tablados de madeira inseridos na atividade $M$, sendo que o tempo para montar um colchão é de aproximadamente 420 segundos. A produção média deste setor, nestas condições é de 57 peças por hora.

Passo 2: Explorar a restrição do sistema - Mesmo verificando-se que seria necessário alterar o layout, (posteriormente na etapa 4) buscou-se explorar esta restrição para identificar outras possíveis oportunidades. Percebeu-se que a montagem dos colchões não era realizada de forma eficiente, pois os funcionários perdiam muito tempo em procurar e selecionar os itens necessários para elaboração do colchão. Utilizando-se de ordens de fabricação, cada abastecedor foi treinado para aumentar o nível de conhecimento dos itens e da rotina estabelecida para função. Neste aspecto, constitui-se uma sequência para o empilhamento dos materiais, de ordem contrária de quem o utiliza, facilitando sua disposição ao uso. Isso resultou em um aumento na produção de peças por hora de 57 para 59, o que representa percentualmente $3,5 \%$.

Não se encontrou outras possibilidades de dedicar esforços nos seguintes itens: redução de setup, pois as máquinas eram ligadas pela manhã e a atividade 
não propunha setups; eliminação de defeitos de fabricação, pois estes não culminavam para restringir o processo; manutenções preventivas eram realizadas em horário alternativo.

Passo 3: Subordinar os demais processos à restrição - Por ser o setor de montagem, esta atividade tem fundamental importância no sistema inteiro, pois está no centro do processo e é sucessora das operações constantes nas atividades $F, E$, I e K na Figura 1. Mesmo assim, o aumento de 3,5\% em peças por hora, não ocasionou gargalos nas atividades anteriores, o que se pode constatar que os demais setores estavam parcialmente ociosos.

Passo 4: Elevar a restrição - Conforme identificado na etapa 1, era visível o desperdício de tempo em tarefas que não agregavam valor, inclusive mais de 15\% do tempo de fabricação era para destinar os produtos à outro setor. Isso se deve à um layout não adequado. O que estava restringindo a atividade no processo eram as distâncias dos materiais, tanto na saída do processo como na entrada dos materiais, pois devido ao espaço limitado alguns funcionários chegavam a percorrer mais de 10 metros para pegar um conjunto de molas (sendo necessários quatro para montar o colchão).

Neste sentido a concepção de um fluxo produtivo eficiente deu-se pela diminuição das distâncias, onde os operadores se deslocassem o mínimo possível. Antes da alteração o processo apresentava uma produtividade de 59 peças por hora e após o novo formato alcançou-se a quantidade de 71 peças por hora (ganho de $20 \%$ ), mesmo com a redução de 3 mesas de montagem e 6 funcionários. Tal fato se deve exclusivamente ao tempo que era perdido em atividades que não agregavam valor. Com apenas 4 mesas restantes a capacidade produtiva foi elevada e os custos com mão de obra reduzidos.

Passo 5: Voltar ao passo um e identificar uma nova restrição - Neste passo constatou-se que a restrição encontrada no sistema foi quebrada. Percebemse mais uma vez que os percentuais de ganho contribuíram para a melhoria contínua de toda organização. Alcançou-se $24 \%$ de aumento em peças por hora no setor, o que é possível afirmar que se obteve aumento no desempenho do processo produtivo por total, de forma que todo o sistema ganhou. Diante disso, para continuar sua evolução, novas análises devem ser feitas para identificar um novo gargalo, retornando ao passo 1. 
Assim, neste trabalho foram utilizadas as ferramentas de cronoanálise para os levantamentos de tempos e identificações das atividades que não agregavam valor. A primeira TOC, realizada no setor de revisão/embalagem foi identificada como gargalo por embalar em média um colchão a cada 84 segundos e de forma manual, sendo estes, os dois fatores responsáveis por prejudicar a capacidade do sistema. Nesta TOC 1, os ganhos na etapa 2 (explorar a restrição do sistema) foram a melhoria na capacidade de produção onde a quantidade de peças por hora aumentou de 43 para 51, equivalente ao percentual de 18,6\%. Mesmo com o avanço o gargalo não foi eliminado e ao dedicar esforços na etapa 4 (elevar a restrição), identificou-se a necessidade de melhorar os equipamentos, o fluxo e o layout do processo. Com base nesta perspectiva a aquisição de uma máquina automática para embalar colchões rendeu um aumento de $41 \%$ na quantidade de peças por hora, em relação a etapa 2. A redução de funcionários de 5 para 3 neste setor também foi fruto das modificações atribuídas ao novo método de trabalho. Desta forma, o gargalo foi eliminado.

Na segunda TOC, uma peça era produzida a cada 63 segundos na atividade L, que era responsável pela montagem dos colchões. Explorou-se o gargalo diminuindo os tempos de preparação atingindo-se um aumento de 3,5\% resultado que é de 59 peças por hora. Na etapa 4 efetuou-se a alteração de layout, principalmente na redução das distâncias entre máquinas e apropriação do fluxo, que melhorou para 71 a quantidade de peças por hora (20,3\%). Conseguiu-se a redução de 17 para 11 funcionários o que representa 35\%. Assim, este gargalo também foi eliminado.

\section{CONCLUSÃO}

A Teoria das Restrições mostrou-se eficaz ao objetivo que se propôs neste trabalho: aumentar o desempenho do processo produtivo. Realizou-se a aplicação dos cinco passos constantes na TOC por duas vezes, sendo a primeira no setor de revisão/embalagem e a segunda na montagem de colchões. Os ganhos adquiridos foram fundamentais para a elevação dos níveis de produtividade, melhorando-se assim o desempenho da unidade fabril. 
Em cada TOC realizada a meta de propor o aumento de desempenho no componente restritivo para que consequentemente acrescente velocidade ao sistema, foi atingida. Neste aspecto é possível verificar principalmente no passo 4 de ambas as TOC, onde o foco foi o de elevar a restrição, seja pela compra de uma máquina ou pela alteração de layout.

O intuito de avaliar os tempos perdidos na restrição, por meio da aplicação da Cronoanálise, com destaque para a identificação de atividades que não geram valor foi concebido, sobretudo na TOC 1 no passo 2, onde designou-se explorar a restrição. Nesta fase do ciclo, explorou-se a atividade em questão de maneira que a taxa de capacidade do recurso aumentou. Esse avanço motivou-se pela tomada de decisão que se possibilitou efetuar, em decorrência do satisfatório levantamento de tempos realizado. Também percebeu-se claramente a intenção de estruturar o processo de focalização das cinco etapas, para gerenciar e controlar as restrições (gargalos) do processo. Todos os passos obrigatórios para contemplar a TOC foram desenvolvidos, aplicados e tiveram resultados positivos.

As conquistas em números possibilitaram que todos os envolvidos da fábrica compreendessem a importância da eliminação das restrições. Já o surgimento de novas restrições de forma sucessiva, mantém o processo de melhoria contínua existente dentro da organização.

Fica evidente que com os resultados obtidos outras melhorias facilmente serão identificadas e ampliadas, pois de fato, se concebeu uma visão crítica perante a importância de ganhar no âmbito global do sistema. Sugere-se a continuidade na identificação de gargalos no setor produtivo de colchões com a construção de uma nova TOC, para que o desempenho seja ainda mais elevado, alcançando-se resultados ainda melhores do que já foram atingidos.

\section{REFERÊNCIAS}

ANDRADE, E. L. Introdução a pesquisa operacional. Rio de Janeiro: LTC, 2014.

BLACKSTONE, John $\mathrm{H}$. Theory of constraints : a status report. International Journal of Production Research, v. 39, n. 6, p.1053-1080, 2001. http://dx.doi.org/10.1080/00207540010028119

BOSCHETTO, S. N. LUDERS, R.; NEVES JR., F.; ARRUDA, L. V. R. Um modelo de otimização da operação de terminais petrolíferos usando a teoria das restrições como préprocessamento. Pesquisa Operacional, v. 29, n.1, p.1-21, 2009. http://dx.doi.org/10.1590/S0101-74382009000100001 
BOYD, L.; GUPTA, M. Constraints management: what is the theory? International Journal of Operations \& Production Management, v. 24, n. 4, p. 370-371, 2004.

http://dx.doi.org/10.1108/01443570410524631

CALIA, R. C.; GUERRINI, F. M. Six Sigma project for scheduling software implementation.

Production, v.15, n. 3, p. 322-333, 2005.

http://dx.doi.org/10.1590/S0103-65132005000300004

CARVALHO, F. S. A ecoeficiência dos processos do sistema de gestão de uma fábrica de colchões e estofados baseado nos suas não conformidades: estudo de caso. 2011. 76f. Dissertação (Mestrado em Tecnologia Ambiental) - Universidade de Santa Cruz do Sul, 2011.

CHIAVENATO, I. Introdução a teoria geral da administração. 7. ed. Rio de Janeiro: Elseiver, 2003.

CONTADOR, José Celso (Coord.). et al. Gestão de Operações: a engenharia de produção a serviço da modernização da empresa. São Paulo: Edgar BlücherLTDA, 1998.

COSTER, I.; FOURNIE, X.; FAURE, C.; ZIANI, E.; NICOLAS, L.; SOUBEYRAND, B.; DAMME, P. V. Assessment of preparation time with fully-liquid versus non-fully liquid paediatric hexavalent vaccines - a time and motion study. Vaccine, v. 33, n. 32, p. 39763982, 2015. http://dx.doi.org/10.1016/j.vaccine.2015.06.030

COX III, J. F.; SPENCER, M. S. Manual da teoria das restrições. Porto Alegre: Bookman, 2002.

DALTON, Michael A. What's Constraining Your Innovation?, Research-Technology Management, v. 52, n. 5, p. 52-64, 2009.

GOLDRATT, E. M.; COX, J. A meta: um processo de melhoria contínua. 2. ed., rev. e ampl. São Paulo: Nobel, c2003.

GUPTA, Mahesh; KLINE, Joseph. Managing a community mental health agency: a theory of constraints based framework. Total Quality Management, v.19, n.3, p. 281-294, 2008.

GUPTA, Mahesh, et al. Improving the weakest link: a TOC-based framework for small businesses. Total Quality Management, v. 21, n. 8, p. 863-883, 2010.

http://dx.doi.org/10.1080/14783360701601850

INMAN, R. A.; SALE, M. L.; GREEN JR, K. W. Analysis of the relationships among TOC use, TOC outcomes, and organizational performance. International Journal of Operations \& Production Management, v. 29, n. 4, p. 341-356, 2009.

http://dx.doi.org/10.1108/01443570910945819

LACHTERMACHER, G. Pesquisa operacional na tomada de decisão. São Paulo: Pearson Prentice Hall, 2009.

LENORT, R.; KLEPEK, R.; SAMOLEJOVÁ, A. Heurisic algorithm for planning and scheduling of forged pieces heat treatment. Metalurgija, v. 51, n. 2, p. 225-228, 2012 ,

LOPETEGUI, M.; YEN, P.Y.; LAI, A.; JEFFRIES, J.; EMBI, P.; PAYNE, P. Time motion 
studies in healthcare: what are we talking about? Journal of Biomedical Informatics, v. 49, p. 292-299, 2014. http://dx.doi.org/10.1016/j.jbi.2014.02.017

MABIN, V. J.; BALDERSTONE, S. J. The Performance of the theory of constraints methodology: analysis and discussion of successful TOC applications. International Journal of Operations and Production Management, v. 23, n. 6, p. 568-595, 2003. http://dx.doi.org/10.1108/01443570310476636

MOREIRA, D. A. Administração da produção e operações. São Paulo: Cengage Learning, 2013.

MOVERGS: Associação das Indústrias de Móveis do Estado do Rio Grande do Sul; Relatório setorial 2014, pólo moveleiro do Rio Grande do Sul. Disponível em: $<$ http://www.movergs.com.br/img/arquivos/movergs/dados-movergs_77.pdf> Acesso em: 1505-2015.

NAOR, M.; BERNARDES, E. S.; COMAN, A. Theory of constraints: is it a theory and a good one? International Journal of Production Research. v. 51, n. 2, p. 542-554, 2013.

http://dx.doi.org/10.1080/00207543.2011.654137

OLIVEIRA, U. R.; MARINS, F. A. S.; ALMEIDA, D. A. Integrating operations management techniques and procedures: an application in a major Brazilian bank. Production, v. 20, n. 2 , p. 237-250, 2010. http://dx.doi.org/10.1590/S0103-65132010005000008

PACHECO, D. A J. Integrando a estratégia de produção com a teoria das restrições, lean e seis sigma: uma abordagem metodológica (Dissertação de Mestrado). Universidade do Vale do Rio dos Sinos, São Leopoldo - RS. 2012.

PACHECO, D. A. J. Teoria das restrições, lean manufacturing e seis sigma: limites e possibilidades de integração. Production, v. 24, n. 4, p. 940-956, 2014.

http://dx.doi.org/10.1590/S0103-65132014005000002

PERGHER, I.; RODRIGUES, L. H.; LACERDA, D. P. Theoretical discussion of the concept of wastes in the Toyota production system: introducing the throughput logic of the theory of constraints. Gestão e Produção, v. 18, n. 4, p. 673-686, 2011.

http://dx.doi.org/10.1590/S0104-530X2011000400001

PRATES, C. C.; BANDEIRA, D. L. Aumento de eficiência por meio do mapeamento do fluxo de produção e aplicação do índice de rendimento operacional global no processo produtivo de uma empresa de componentes eletrônicos. Gestão e Produção. v.18, n.4, p. 705-718, 2011.http://dx.doi.org/10.1590/S0104-530X2011000400003

PRETORIUS, Pieter. Introducing in-between decision points to TOC's five focusing steps. International Journal of Production Research, v. 52, n.2, p. 496-506, 2014. http://dx.doi.org/10.1080/00207543.2013.836612

QUELHAS, O.; BARCAUI, A. B. A teoria das restrições aplicada a gerência de projetos: uma introdução à corrente crítica. [2008]. Disponível em:

<www.pmtech.com.br/newsletter/Marco_2005/TOC_e_CCPM_em_GP.pdf >. Acesso em: 11 nov. 2016.

RITZMAN, L. P.; KRAJEWSKI, L. J. Administração da produção e operações. São Paulo: Pearson Prentice Hall, 2004. 
SANTOS, A. R. Metodologia científica: a construção do conhecimento. 4. Ed. Rio de Janeiro: DP\&A, 2000.

SLACK, N.; CHAMBERS, S.; JOHNSTON, R. Administração da produção. 2. ed. São Paulo: Atlas, 2002.

SOBREIRO, V. A.; NAGANO, M. S. Um novo método heurístico para a otimização de mix de produção baseado na teoria das restrições e no problema da mochila. Revista Produção Online, v. 13, n.2 p. 520-543, 2013. http://dx.doi.org/10.14488/1676-1901.v13i2.1125

SOUZA, F. B. From the OPT to the theory of constraints: advances and myths. Production, v. 15, n. 2, p. 184-197, 2005. http://dx.doi.org/10.1590/S0103-65132005000200005

SUGAI, M. Avaliação do uso do MTM (Methods-Time Measurement) em uma empresa de metal-mecânica. 2003. 115 f. Dissertação de mestrado (Mestre em Engenharia Mecânica) - Faculdade de Engenharia Mecânica, Universidade Estadual de Campinas, Campinas, 2003.

THIOLLENT, M. J. M. Metodologia da pesquisa-ação. São Paulo: Cortez, 2008.

TOLEDO JUNIOR, I. F. B. Cronoanálise. 13. ed. Mogi das Cruzes: O\&M Itys-Fides, 1995. 205 p. (Série Racionalização industrial)

WATSON, K. J.; BLACKSTONE, J. H.; GARDINER, S. C. The evolution of a management philosophy: the theory of constraints. Journal of Operations Management, n. 25, n. 2, p. 387-402, 2007. http://dx.doi.org/10.1016/j.jom.2006.04.004

WU, H. H.; LEE, A. H. I.; TSAI, T. P. A two-level replenishment frequency model for TOC supply chain replenishment systems under capacity constraint. Computers \& Industrial Engineering, v. 72, p. 152-159, 2014. http://dx.doi.org/10.1016/j.cie.2014.03.006

WU, H. H.; CHEN, C. P.; TSAI, C. H.; TSAI, T. P. A study of an enhanced simulation model for TOC supply chain replenishment system under capacity constraint. Expert Systems with Applications, v. 37, n. 9, p. 6435-6440, 2010. http://dx.doi.org/10.1016/j.eswa.2010.02.074

ZIVALJEVIC, A. Theory of constraints - application in land transportation systems.

Management of Environmental Quality: An International Journal, v. 26, n. 4, p. 505-517, 2015. http://dx.doi.org/10.1108/MEQ-07-2014-0110

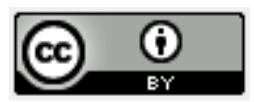

Artigo recebido em 15/01/2016 e aceito para publicação em 15/12/2016 DOI: http://dx.doi.org/ 10.14488/1676-1901.v17i1.2321 